\title{
Flow-rate fluctuations in the outpouring of grains from a two-dimensional silo
}

\author{
A. Janda, ${ }^{1, *}$ R. Harich, ${ }^{2}$ I. Zuriguel, ${ }^{1}$ D. Maza, ${ }^{1}$ P. Cixous, ${ }^{2}$ and A. Garcimartín ${ }^{1}$ \\ ${ }^{1}$ Departamento de Física, Facultad de Ciencias, Universidad de Navarra, E-31080 Pamplona, Spain \\ ${ }^{2}$ Laboratoire de Physique et Mécanique des Milieux Hétérogènes, associé au CNRS (UMR 7636) et aux Universités Paris 6 et Paris 7 , \\ 10 rue Vauquelin, 75005 Paris, France
}

(Received 12 November 2008; published 18 March 2009)

\begin{abstract}
We present experimental results obtained with a two-dimensional silo discharging under gravity through an orifice at the flat bottom. High-speed measurements provide enough time resolution to detect every single bead that goes out and this allows the measurement of the flow rate in short-time windows. Two different regimes are clearly distinguished: one for large orifices, which can be described by Gaussian fluctuations, and another for small orifices, in which extreme events appear. The frontier between those two regimes coincides with the outlet size below which jamming events are frequent. Moreover, it is shown that the power spectrum of the flow-rate oscillations is not dominated by any particular frequency.
\end{abstract}

DOI: 10.1103/PhysRevE.79.031302

PACS number(s): $45.70 . \mathrm{Mg}$

\section{INTRODUCTION}

Granular media often reveal their characteristic features in dense flows $[1,2]$. One particular situation where they are displayed is in the passage of grains through an obstacle, such as a throttle or an orifice. Silo discharge is the archetypal example where this situation can be observed. Indeed, a lot of technological efforts have been invested to control this process, and specifically to stabilize the flow during the discharge process. As finely divided solids can form arches, the flux of particles may be interrupted by the appearance of such a structure, which is capable of supporting the weight above. Even when arches are not stable and the flow is not completely arrested, these structures can introduce strong fluctuations in the mass delivery and thus induce many practical problems.

Previous results [3-11] have revealed that, in silos of two and three dimensions, the formation of an arch that causes a jam (i.e., a definitive halt of the flow) strongly depends on the size of the exit orifice. It is still unclear whether there is a critical value for the orifice, in the sense that a phase transition takes place at that point $[7,12]$. If this were the case, such a value would establish a frontier between two distinct regimes: one for small orifices, where jamming would be unavoidable (provided that one is able to wait a sufficient time) and the other for large orifices, in which jamming would never happen even if one waits for an infinite time. Even if this value is not critical in the evoked sense, there are compelling evidences that jamming is common for small orifices, and rather unusual for big ones. The frontier between both situations is quite well defined and it is expected that also the flow rate (the number of beads fallen per unit time) displays different properties at each regime. Indeed, in a previous work Longhi et al. [13] reported different behavior of the force fluctuation during the discharge of a silo through large and small orifices.

It is well established that the mean flow rate of grains through the exit of a three-dimensional (3D) silo depends on

\footnotetext{
*ajandaga@alumni.unav.es; http://fisica.unav.es/granular/
}

the diameter of the orifice as $D^{2.5}[14,15]$ if the hole is large. In the same way, in a two-dimensional (2D) silo, the mean flow is a function of $D^{1.5}$ as shown in a recent work [16]. In this work, important deviations from this behavior have been reported for small orifices in both two- and threedimensional silos. However, the mean flow rate does not display any abrupt change at any particular size of the outlet. It should be noted that in the aforementioned works the term "mean flow rate" refers to the average during a given lapse of time. Indeed, it has been found that, during the avalanche, the flow rate is not constant as it fluctuates around its mean value [17-19]. However, there is a lack of knowledge about the features of the flow-rate fluctuations and their dependence on the size of the outlet, yet it is reasonable to expect different behavior for the two mentioned regimes. Our aim in this paper is to study systematically the flow fluctuations at the outlet orifice.

Besides, about 50 years ago Brown and Richards [20] speculated about the existence of periodic density oscillations - which they called dilatant waves-with a frequency around $2 \mathrm{~Hz}$ in the discharge of a silo through small orifices. These results suggest the existence of flow-rate oscillations. Nevertheless, the crudeness of their method (as judged by modern standards) did not allow them to support their assessment conclusively. To our knowledge, this issue has not been revisited experimentally.

In this paper we present an experiment that allows the detection of individual grains at the outlet of a silo. For this purpose it is necessary to have a very high temporal resolution, as the moment in which each particle passes through the outlet must be measured with high precision. This has been carried out with a $2 \mathrm{D}$ silo where we take pictures of the outlet region at high rate in order to track the particles. The experimental methods will be explained in the following section. We will then proceed to describe the statistical properties of the flow rate, first as calculated by taking the number of beads fallen during a certain time window, and then by means of a related quantity which is the time elapsed between two successive beads. In the last section, we will summarize our conclusions. 


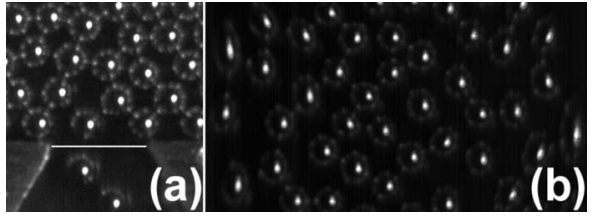

FIG. 1. (a) Photograph of the outlet orifice. Each bead gives a bright spot that allows particle tracking. The superimposed white line shows the place where the pixels are acquired. (b) Space-time diagram. The horizontal span of the image is $10 \mathrm{~mm}$, and the vertical one $15 \mathrm{~ms}$.

\section{EXPERIMENTAL METHODS}

In order to carry out the experiments reported in this paper, we have used a two-dimensional silo made of two glass panes spaced by two steel stripes. The thickness of these stripes $(1.1 \mathrm{~mm})$ determines the gap where the grains are stocked, and the distance between them set the width of the silo (which is 200 times the diameter of the beads). We have used stainless-steel spheres of diameter $d=1.00 \pm 0.01 \mathrm{~mm}$, which warrant the formation of a monolayer of particles. The bottom of the silo is flat and is made of two movable stainless-steel blades, forming the exit orifice [see Fig. 1(a)]. The size of the exit orifice is adimensionalized by dividing its length $D_{0}$ by the diameter of the beads: $D=D_{0} / d$. When the size of the outlet is large enough, the beads pour freely from the silo due to gravity. However, if the size of the orifice does not exceed a few bead diameters, the flow can be completely and permanently arrested due to the formation of an arch that provokes a jam. We define avalanche as the discharge of grains that takes place between two successive jams. The setup has already been described in another paper [12]; for further information, we refer the interested reader to it.

Flow-rate measurements are performed by taking a film of the outlet with a high-speed camera (Photron Fastcam 1024). Under convenient illumination, each bead will reflect a white spot that can be easily tracked [Fig. 1(a)]. Then the vertical velocity $v_{z}$ of every particle can be obtained when it passes through the outlet, that is, when it crosses the white line of pixels represented in Fig. 1(a). From these data the velocity profile along the horizontal direction can be obtained. This is carried out by slicing the horizontal line marking the outlet in small segments of the same length and averaging the velocities of the particles that cross the outlet at each one of them. In Fig. 2 the velocity profile is presented for a silo with an outlet of $D=10$, where the averages were performed for segments of $0.15 d$. It is evident that there is an important gradient of velocity at the outlet, especially near the borders.

Particle tracking, as explained above, can provide the moment at which a particle passes through the outlet. However, this technique does not offer a good temporal resolution and is time consuming in terms of computer analysis. As in this work we are only interested in the instant at which each particle passes through the outlet; a different technique has been implemented in order to increase the data volume and save computing time. The procedure is to acquire a single line of pixels spanning the opening. This line of pixels is

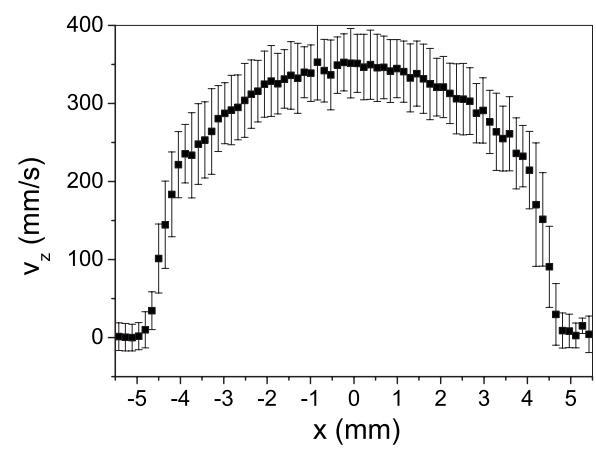

FIG. 2. Vertical velocity profile along the horizontal direction at the outlet of a silo [the white line of pixels in Fig. 1(a)] for $D$ $=10$. Error bars correspond to the standard deviation of the measurements.

stacked at the camera sampling rate, forming an image where the vertical dimension is time and the horizontal one is distance [an example is provided in Fig. 1(b)]. Speeds of the films reported here ranged from 3000 to 18000 frames per second, which is large enough to measure the time elapsed between the passage of two consecutive beads. Image processing can yield subpixel resolution when locating the centroids of the bright spots appearing in images such as the one displayed, so the temporal resolution attained was better than $3 \times 10^{-5} \mathrm{~s}$.

These measurements provide the moments at which the center of every bead passed through the line depicted in Fig. 1(a). From these data, we can define the flow rate $q$ as the number of beads that come out from the orifice during a fixed interval of time. Note that for the data reported in this paper these time intervals do not overlap. The time window we have taken was chosen so as to include a significant number of beads (an average of more than ten grains in the worst case) for this magnitude to make sense. In particular, the value for many of the measurements performed is 30 $\times 10^{-3} \mathrm{~s}$, which is larger than the time that a bead takes to fall on its own diameter under the action of gravity, i.e., $\sqrt{\frac{2 d}{g}} \approx 14 \times 10^{-3}$ s. In Fig. 3 we show examples of the measurement of $q$ for two different outlet sizes. We have also measured the time interval between two consecutive grains, $\Delta t_{i}$, which is the time elapsed between the passage of the $i$ -1 and the $i$ th beads through the orifice.

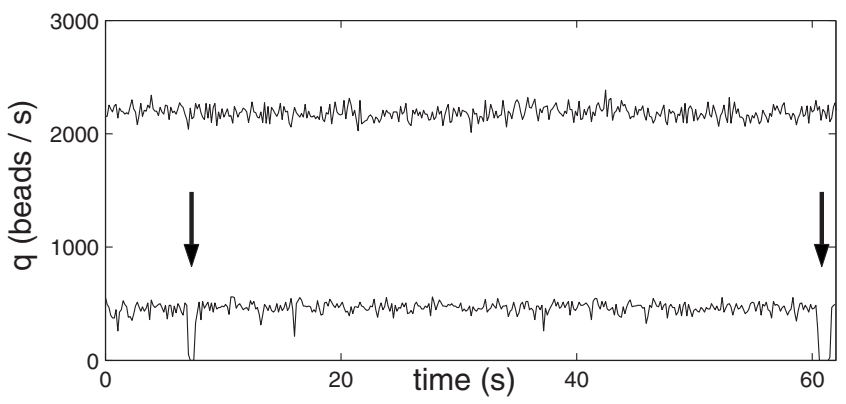

FIG. 3. The flow rate $q$ (beads per unit time as measured at nonoverlapping time windows of $150 \mathrm{~ms}$ ) for an orifice size $D$ $=9.5$ (top) and $D=4.3$ (bottom). The arrows mark two events where the flow has ceased for a time longer than the window. 
All of the measurements were taken during the discharge process, after waiting for $5 \mathrm{~s}$ subsequently to resuming the outpouring, in order to avoid the transient flow regime that has been observed at the beginning of the avalanche [17-19]. It is important to remark that for small orifices several avalanches must be measured in order to get a significant amount of data. In these cases, the flow is resumed breaking the arch, which blocks the outlet, by means of a jet of pressurized air aimed at the orifice from beneath.

\section{FLUCTUATIONS OF THE FLOW RATE}

A salient feature of the flow rate $q$ is revealed in Fig. 3 . For small orifices (what small means in this context will be qualified later), fluctuations look similar to those appearing for big orifices, but they include short-time intervals in which the flow goes to zero and other downward spikes where the flow rate slows down significantly. In the case displayed in Fig. 3, for instance, two events are visible in which the flow was virtually zero for a time longer than the window used to calculate $q$. These temporary interruptions of the flow are presumably related to arches that block the exit orifice but are either unstable or unable to obstruct the entire orifice. No energy input whatsoever is needed to restart the flow, and grain outpouring resumes by itself. Sometimes these flow interruptions are not complete, and the flow falls abruptly without ceasing completely. It should also be noted that the time window chosen to calculate $q$ imposes a cutoff for the detection of such temporary jamming events. If the time window is large, then these events can manifest themselves in spikes that do not attain strictly a zero value but a small one. We suspect that some of the spikes seen in Fig. 3 are just that. The flow-rate fluctuations displayed for $D$ $=4.3$ suggest that extreme events of low $q$ are probable but events of large $q$ are not observed. In other words, the fluctuations for small orifices are not symmetrically distributed.

A better description of the features of the flow rate can be obtained by calculating the normalized histograms of the values of $q$ obtained for different sizes of the exit aperture. These results are displayed in Fig. 4 revealing that for large orifices the fluctuations are well fitted by a Gaussian. On the contrary, for sizes of the orifice below $D=7.0$ the fluctuations do not conform to a Gaussian anymore. Indeed it can be observed that even for $D=7.0$ the Gaussian fit is shifted a little bit to the left. This fact is easily appreciated if one focuses on the maximum of the curve. For sizes of the orifice below $D=7.0$, the histograms seem to be the combination of a Gaussian with another set of events at or near $q=0$.

It is interesting to note that the presence of events at $q$ $=0$ occurs only for small values of $D$, just in the region where it has been found that jamming is frequent (with greater and greater probability as the orifice gets smaller). On the contrary, for high values of $D$-where the probability of observing a jam that completely stops the flow is very lowthere is no event at $q=0$. Indeed, the value of $D$ at which the events at $q=0$ disappear coincides approximately with the region at which the jamming probability for a large number of beads decreases abruptly to zero [12]. According to this work, there is not a critical value of $D$ above which jamming
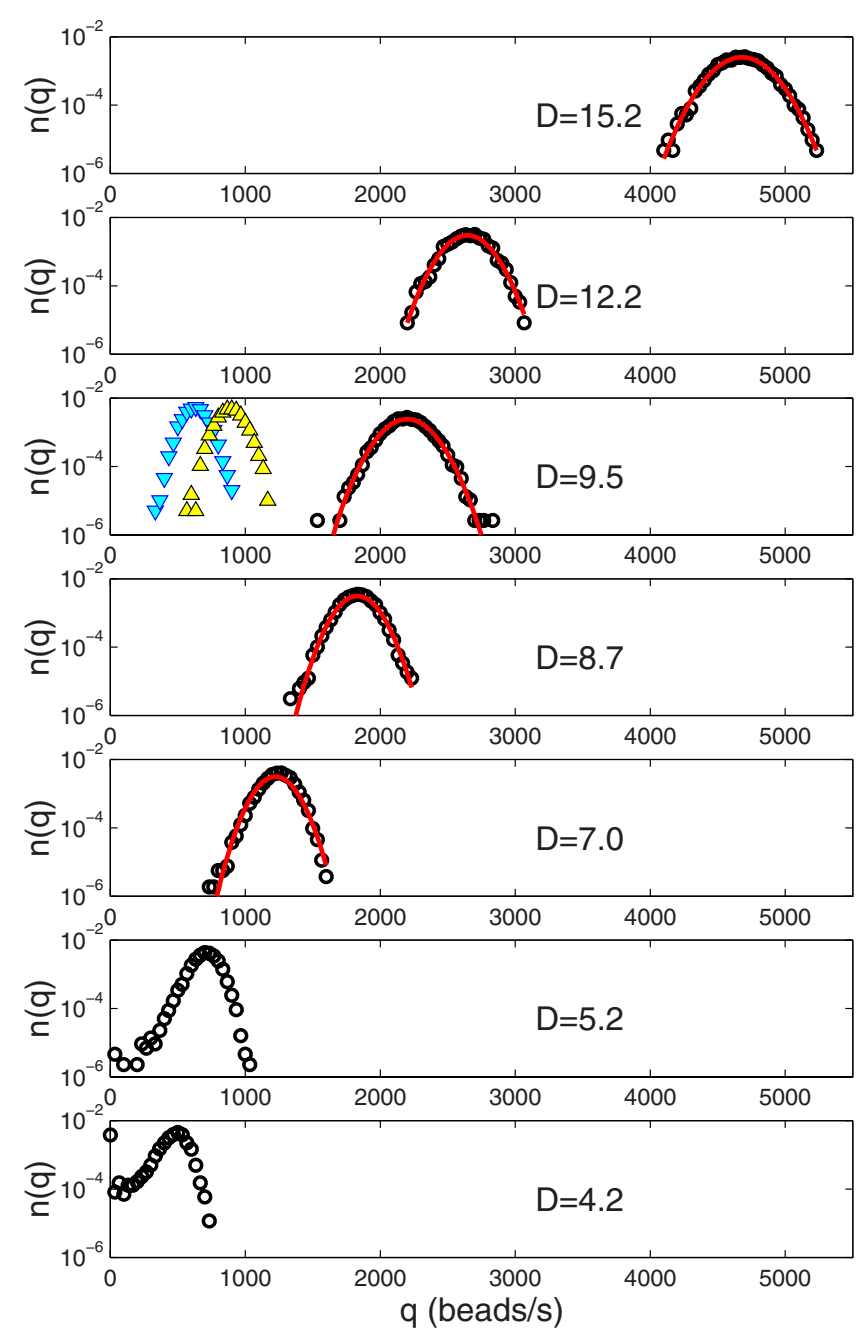

FIG. 4. (Color online) Normalized histograms of the flow rate $q$-calculated as the number of beads fallen within a time window of $30 \mathrm{~ms}$ - for different outlet sizes $(D)$, as indicated in the plots. The red solid line is a Gaussian fit presented only for $D \geq 7.0$. Down and up triangles are histograms obtained for $D=9.5$ taking into account only the beads that crossed the orifice closer than 2.1 $\mathrm{mm}$ to the edges or closer than $4.2 \mathrm{~mm}$ to the left edge, respectively.

is not possible, as the position of the transition moves to higher values of $D$ when the number of particles used to build the jamming probability is increased. This fact implies that events at $q=0$ should exist even for the largest $D$. However, these events would be so rare for large outlet orifices that they would be observed only if the flow fluctuations were measured during exceedingly long times.

One could consider the possibility that the blockages could be a wall effect that takes place near the edges of the orifice. If this were the case, for large orifices the zero flow events near the edges would be masked by the continuous flow in the central zone of the orifice. But then the flow near the walls, even for large orifices, should also display events near $q=0$. We have obtained histograms just taking into account the grains that crossed the orifice nearer than $2.1 \mathrm{~mm}$ from the edges for $D=9.5$ so that one can compare with the orifice $D=4.2$. We have also taken the histogram for the beads that crossed the orifice closer than $4.2 \mathrm{~mm}$ to the left 

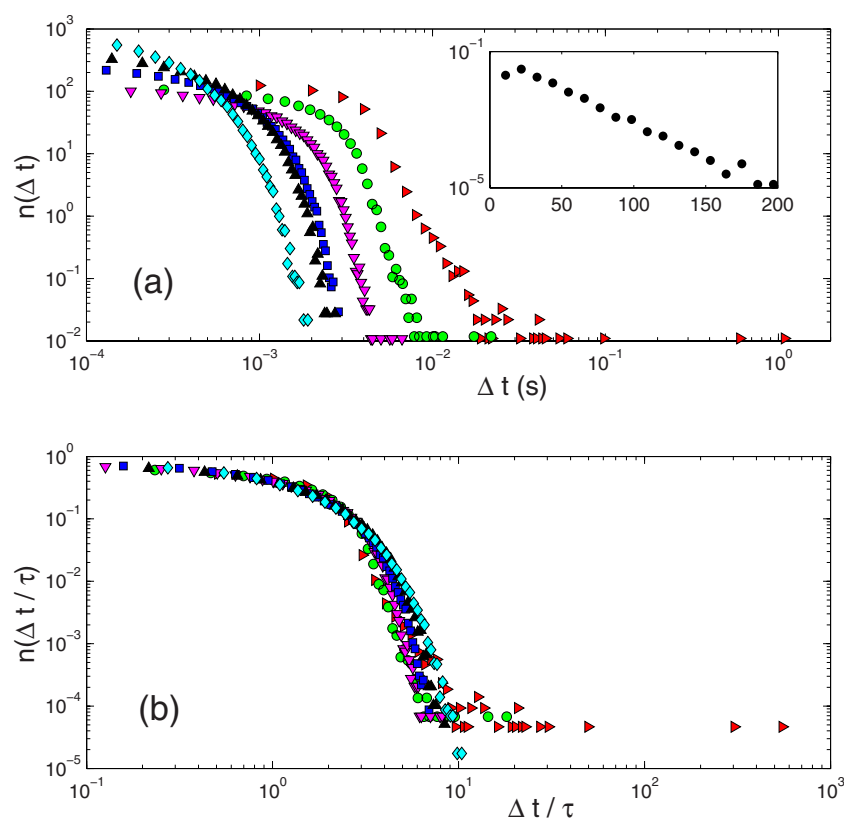

FIG. 5. (Color online) (a) Histograms of $\Delta t_{i}$ for different $D$, in logarithmic scale. Symbols correspond to the following values of the exit orifice: diamonds, $D=15.5$; up triangles, $D=10.9$; squares, $D=9.5$; down triangles, $D=7.0$; circles, $D=5.3$; right triangles, $D$ =4.3. Inset: the histogram obtained for $D=15.5$ taking only the beads that exit the orifice through a segment of a length $0.4 d$ around the center. Note that in the inset the horizontal scale is linear and the vertical one is logarithmic. (b) Normalized histograms for $\Delta t_{i}$ [same data as in (a)] rescaled with the mean rate $\tau$ (i.e., the average time interval between grains).

edge. Both histograms are shown with different symbols in Fig. 4 and do not display a shape similar to the histograms for small orifices. As such, we can conclude that these events are not "wall effects" in the sense mentioned above.

\section{TIME INTERVAL BETWEEN GRAINS}

We can take a closer look into the temporary interruptions of the flow by calculating the statistical properties of the time interval between the passing of consecutive beads, which we call $\Delta t_{i}$. The inverse of this quantity has the dimension of a frequency and could be used to define an "instantaneous flow rate" in units of beads per time, which could be calculated as one bead divided by the time elapsed until the next bead passes through the outlet. But this variable would be sampled only when a bead crosses the exit line, and as these events are not regular (i.e., evenly spaced), the probability distributions would be biased. It is more apt to look at the histograms for the time intervals between beads $\left(\Delta t_{i}\right)$ which are presented in Fig. 5. Since the probability of passing without jamming of consecutive grains has been proposed to be independent events as a first approximation $[6,12]$, one could naively expect an exponential shape in the $\Delta t_{i}$ distribution resulting from a Poisson process. This is indeed the case if one restricts the observation to a narrow zone of the exit orifice. For instance, the histogram of $\Delta t_{i}$ obtained in a region of $0.4 d$ at the center of an outlet of size $D=15.5$ is presented in the inset of Fig. 5(a). The same kind of distributions is obtained for any narrow region at any given position of the outlet. The only difference for the exponential distributions at different positions is the value of the average $\Delta t_{i}$. This is just a consequence of the fact that the mean vertical velocity $\left(v_{z}\right)$ changes along the outlet as displayed in Fig. 2. As a result, when the region where the $\Delta t_{i}$ are measured includes all the orifice, the superposition of many such exponential distributions with different mean $\Delta t_{i}$ gives rise to the probability density function (PDF) shown in Fig. 5(a).

When the $\Delta t_{i}$ histograms of the whole orifice are compared for different values of $D$, it becomes evident a clear difference in their shape for large and small $D$. For $D>7$ there is a well defined cutoff value for $\Delta t_{i}$, meaning that it is very unlikely to find a time interval longer than the cutoff in which no bead goes through the exit. On the contrary, for small orifices there are a number of events that are by no means negligible beyond the cutoff value. Indeed the smaller the orifice the higher the number of these extreme events. This is more clearly seen if all the histograms are rescaled by dividing the horizontal axis by the inverse of the mean flow rate and normalizing the values so that the area below the curve is one [Fig. 5(b)]. In this case all the histograms match except for the extreme events that correspond to large time intervals during which no bead has crossed the exit. This is an alternative way of interpreting the results of the flow-rate histograms shown in Fig. 4. It can be observed that the region of small exit orifices, where extreme events appear for $q$ near or equal to zero, coincides with the values of $D$ for which the histograms of $\Delta t_{i}$ display a significant number of events beyond the cutoff value.

\section{OSCILLATIONS OF THE FLOW RATE}

Let us now present the results of the investigation about the existence of periodic oscillations in the flow rate at the exit orifice. In 1964, Brown and Richards [20] reported that their data hinted at the existence of oscillations in the packing fraction in a region near the outlet with a frequency around $2 \mathrm{~Hz}$. Hence, it may be thought that this could lead to oscillations of the same frequency in the outflow. Veje and Dimon [21] have studied experimentally the flow in a smallangle funnel mounted on a slightly inclined plane. Shock waves appeared inside the funnel when its angle was small but they did not find any evidence of periodic oscillations when the funnel was straight. In an hour glass [22] oscillations are indeed present but this is due to the influence of the air flow percolating through the fine sand in a small orifice. To our knowledge, this subject has not been revisited in a silo, as in the experiment of Brown and Richards. We have calculated the power spectrum of the flow rate $q$. Here we have taken a time window of $130 \mathrm{~ms}$ to calculate $q$ because it is the smallest one that can be consistently used for all the cases considered. As can be seen in Fig. 6, the spectrum is flat in all the cases up to the maximum frequency that can be measured, which is about $5 \mathrm{~Hz}$ (larger, in any case, than the frequency of approximately $2 \mathrm{~Hz}$ put forward by the above mentioned authors). The flat power spectrum is a strong evidence against the existence of low-frequency oscillations at 


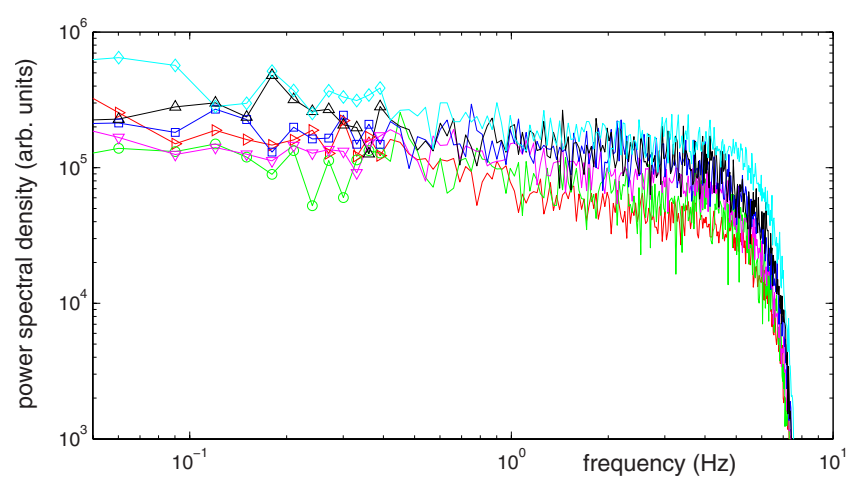

FIG. 6. (Color online) Power spectrum of the flow-rate fluctuations, measuring the flow in time windows of $130 \mathrm{~ms}$. Symbols correspond to the following values of the exit orifice: diamonds, $D=15.5$; up triangles, $D=10.9$; squares, $D=9.5$; down triangles, $D=7.0$; circles, $D=5.3$; right triangles, $D=4.3$. For the sake of clarity, symbols are displayed only at the left part of the plots.

the exit of a flat-bottomed two-dimensional silo. In the future it should be checked that this result is also valid in the threedimensional case.

\section{CONCLUSIONS}

In this work we have studied the flow rate through an orifice at the bottom of a two-dimensional silo discharging by gravity. The resolution attained allows the detection of individual beads coming out from the exit orifice and the measurement of the flow rate in short-time windows with high accuracy.

We have first shown that the fluctuations of the flow, calculated in a short temporal scale, display different properties above and below a certain size of the orifice. This value, called $D_{c}$, was previously identified as the frontier between two flow regimes: for $D<D_{c}$ jamming can occur while for $D>D_{c}$ jamming is rather unlikely in a finite amount of time. Correspondingly, flow-rate fluctuations are Gaussian above $D_{c}$ but not below it. For small apertures, temporary interruptions of the flow become statistically relevant and these events break down the Gaussian shape. The same events can be seen if the time interval between consecutive grains is considered: extreme events (meaning large time intervals far from a well defined cutoff value, in which no bead falls) appear for small sizes of the orifice. Moreover, the investigation of the power spectrum of the flow-rate oscillations has revealed that there is not any particular frequency in the outflow.

\section{ACKNOWLEDGMENTS}

Eric Clément has contributed to the ideas developed in this paper in many discussions, and we gratefully acknowledge his support. We also want to thank M. Pastor, C. Mankoc, and E. Kolb for their comments. This work was funded by the Spanish Government (Projects No. FIS2005-03881 and No. FIS2008-06034-C02-01) and in particular by Acción Integrada Contract No. HF 2006-0234 and Egide-PHCPicasso 2007 (French Government). A.J. thanks Fundación Ramón Areces for support.
[1] G. D. R. Midi, Eur. Phys. J. E 14, 341 (2004).

[2] R. M. Nedderman, Statics and Kinematics of Granular Materials (Cambridge University Press, Cambridge, 1992).

[3] H. Sakaguchi, E. Ozaki, and T. Igarashi, Int. J. Mod. Phys. B 7, 1949 (1993).

[4] K. To, P. Y. Lai, and H. K. Pak, Phys. Rev. Lett. 86, 71 (2001).

[5] I. Zuriguel, L. A. Pugnaloni, A. Garcimartín, and D. Maza, Phys. Rev. E 68, 030301 (2003).

[6] I. Zuriguel, A. Garcimartín, D. Maza, L. A. Pugnaloni, and J. M. Pastor, Phys. Rev. E 71, 051303 (2005).

[7] K. To, Phys. Rev. E 71, 060301(R) (2005).

[8] E. Clément, G. Reydellet, F. Rioual, B. Parise, V. Fanguet, J. Lanuza, and E. Kolb, in Traffic and Granular Flow '99, edited by D. Helbing, H. J. Herrmann, M. Schreckenberg, and D. E. Wolf (Springer, Berlin, 2000), pp. 457-468.

[9] L. A. Pugnaloni and G. C. Baker, Physica A 337, 428 (2004).

[10] A. Mehta, Granular Physics (Cambridge University Press, Cambridge, 2007).

[11] G. Pérez, Pramana, J. Phys. 70, 989 (2008).
[12] A. Janda, I. Zuriguel, A. Garcimartín, L. A. Pugnaloni, and D. Maza, Europhys. Lett. 84, 44002 (2008).

[13] E. Longhi, N. Easwar, and N. Menon, Phys. Rev. Lett. 89, 045501 (2002).

[14] W. A. Beverloo, H. A. Leniger, and J. Van de Velde, Chem. Eng. Sci. 15, 260 (1961).

[15] R. M. Nedderman, U. Tüzün, S. B. Savage, and G. T. Houlsby, Chem. Eng. Sci. 37, 1597 (1982).

[16] C. Mankoc, A. Janda, R. Arévalo, J. M. Pastor, I. Zuriguel, A. Garcimartín, and D. Maza, Granular Matter 9, 407 (2007).

[17] G. H. Ristow, Physica A 235, 319 (1997).

[18] Y. Xu, K. D. Kafui, C. Thornton, and G. Lian, Part. Sci. Technol. 20, 109 (2002).

[19] S. C. Yang and S. S. Hsiau, Powder Technol. 120, 244 (2001).

[20] R. L. Brown and J. C. Richards, Trans. Inst. Chem. Eng. 38, 243 (1960).

[21] C. T. Veje and P. Dimon, Phys. Rev. E 54, 4329 (1996).

[22] X-1. Wu, K. J. Maloy, A. Hansen, M. Ammi, and D. Bideau, Phys. Rev. Lett. 71, 1363 (1993). 